\title{
Quadratic divergences in gauge theories
}

\author{
M.S. Al-sarhi, I. Jack, D.R.T. Jones \\ DAMTP, University of Liverpool, P.O. Box 147, Liverpool L69 3BX, UK
}

Received 20 January 1992; in revised form 6 March 1992

\begin{abstract}
Quadratic divergences are analysed using dimensional regularisation in gauge theories in general and the standard model in particular. We give a prediction (under dubious assumptions) that $m_{t} \approx 115 \mathrm{GeV}$ and $m_{H} \approx 180 \mathrm{GeV}$.
\end{abstract}

\section{Introduction}

There has been some interest recently in the significance of the quadratic divergences generally present in renormalisable quantum field theories, especially in the context of dimensional regularisation. In dimensional regularisation, quadratic divergences manifest themselves as poles at unphysical values of the spacetime dimension $(d)$ [1]. Following Veltman's work, various authors [2-13] explored the structure of quadratic divergences in theories in general and in the standard model in particular. Supersymmetric theories (without $U(1) D$-terms) are free of quadratic divergences, and one motivation for this work was the search for non-supersymmetric theories free of quadratic divergences $[2,3,7]$. The standard model, in fact, may be made so at one loop by imposing a single relationship between the top $\left(m_{t}\right)$ and Higgs $\left(m_{H}\right)$ masses [1]. In [7] we gave the two loop result for a general gauge theory, and mentioned that in the standard model case the result gives a condition on $m_{t}$ and $m_{H}$ incompatible with Veltman's one loop constraint. We also observed a remarkable relationship between the one and two-loop conditions in the general case, based on renormalisation group dependence of the one-loop constraint. We subsequently pursued possible extensions to higher loops, but in non-gauge theories only $[9,10]$. We restricted ourselves to non-gauge theories partly for reasons of simplicity, but mainly because the treatment of gauge theories presents special problems. Our main purpose here is to elucidate these problems. Because other authors have considered $[8,12]$ the special case of the standard model, we feel it worthwhile presenting our results in more detail in this case. While there is definitely no simultaneous solution to the one and two loop constraints [7], we show that requiring scale invariance of the one loop constraint leads to the "prediction" $m_{t} \approx 115 \mathrm{GeV}$ and $m_{H} \approx 180 \mathrm{GeV}$ if strong interaction effects are ignored.

Our calculations reveal that dimensional regularisation and dimensional reduction lead to qualitatively different results, which raises the question of the universality of quadratic divergences. In the Conclusions we suggest investigating this further by rederiving the results using an entirely different regulator, such as non-local regularisation [14].

\section{Non-gauge theories}

Within dimensional regularisation, the poles in $d$ that characterise quadratic divergences in renormalisable theories in four dimensions occur at [1] $d=4-2 / L^{\prime}$ where $L^{\prime} \in \mathbb{N}$ and $L^{\prime} \leq L$ and $L$ is the number of loops. Thus at one loop there are poles at $d=2$, and at two loops poles at $d=2$ and $d=3$, and so on. We refer to the pole corresponding to $L^{\prime}=L$ (at $L$ loops) as the leading pole; the others can be obtained from lower order calculations via renormalisation group identities. In [7] we calculated the residue of the leading pole $(d=3)$ at $L=2$ for a general renormalisable gauge theory with scalars and fermions. We observed a remarkable relationship between this result and the one loop $(d=2)$ residue. In a theory with scalars and fermions only, with Lagrangian given by

$L=\frac{1}{2}\left(\partial_{\mu} \Phi\right)^{2}+i \Psi \sigma \cdot \partial \bar{\Psi}-U(\Phi)-\frac{1}{2}[\Psi M(\Phi) \Psi+$ c.c. $]$

where $M=m+Y_{a} \Phi_{a}$ and $U(\Phi)$ is a quartic polynomial, this relationship takes the form [up to an overall constant]

$\Delta_{2}=\left[\beta_{U}^{(1)} \cdot \frac{\partial}{\partial U}+\beta_{M}^{(1)} \cdot \frac{\partial}{\partial M}\right] \Delta_{1}-\Delta_{1} \cdot \frac{\partial}{\partial U} \beta_{U}^{(1)}$.

Here $A_{L}$ represents the $L$ loop leading pole residue. The one-loop $\beta$-functions $\beta_{U}^{(1)}$ and $\beta_{M}^{(1)}$ are given by

$\beta_{U}^{(1)}=\frac{1}{2} U_{a b} U_{a b}+\frac{1}{2}\left[U_{a} \operatorname{tr}\left(Y_{a} Y_{b}^{*}\right) \Phi_{b}+\right.$ c.c. $]-\operatorname{tr}\left(M M^{*} M M^{*}\right)$ 
and

$$
\begin{aligned}
\beta_{M}^{(1)}= & 2 Y_{a} M^{*} Y_{a}+\frac{1}{2}\left(Y_{a} Y_{a}^{*} M+M Y_{a}^{*} Y_{a}\right) \\
& +\frac{1}{2} Y_{a} \Phi_{b} \operatorname{tr}\left(Y_{a} Y_{b}^{*}+Y_{a}^{*} Y_{b}\right)
\end{aligned}
$$

where $U_{a}=\partial \mathrm{U} / \partial \Phi_{a}$ etc. $\Delta_{1}$ and $\Delta_{2}$ are given in (4.2) and (4.4) of [8]. It is easy to verify that (2.2) holds (up to an overall constant).

Requiring $\Delta_{2}=\Delta_{1}=0$ is equivalent to requiring $\Delta_{1}=\frac{\partial}{\partial \mu} \Delta_{1}=0 ;$ in other words that the one loop condition for absence of quadratic divergences be scale invariant. At $L=3$ we found [8] a relationship analogous to (2.2); at $L=4$ we were unable to discover such a relationship [9], at least using $\beta$-functions defined using minimal subtraction. It is possible, however, that such a relationship does exist if $\beta$-functions defined according to some other scheme are employed. We do not pursue this idea here, but instead return to gauge theories, where subtleties occur even at lowest order.

\section{Gauge theories}

We consider a general renormalisable gauge theory, with Lagrangian

$$
\begin{aligned}
L= & -\frac{1}{4} G_{\mu \nu}^{2}+i \Psi \sigma \cdot D \bar{\Psi}+\frac{1}{2}\left(D_{\mu} \Phi\right)^{2}-U(\Phi) \\
& -\frac{1}{2}[\Psi M \Psi+\text { c.c. }]
\end{aligned}
$$

where $\Phi, \Psi$ transform according to gauge group representations $\Theta^{A}, t^{A}$ respectively:

$\left[\Theta^{A}, \Theta^{B}\right]=i f^{A B C} \Theta^{C}, \quad\left[t^{A}, t^{B}\right]=i f^{A B C} t^{C}$.

The one loop residue at $d=2$ is given (up to an overall constant) by the expression [7]

$\Delta_{1}=(n-1) g^{2} \Phi^{T} \Theta^{A} \Theta^{A} \Phi+U_{a a}-2 \operatorname{Tr}\left(M M^{*}\right)$

where $g$ is the gauge coupling.

In (3.2), $n$ stands for the trace of the metric tensor: it arises from the "seagull" contribution to the scalar selfenergy and consequently is the dimensionality of the $\mu$ index of the vector field $W_{\mu}$. The question arises as to whether it should be set equal to 4 or to 2 . This problem was addressed by Veltman [1] in his original discussion of quadratic divergences in the context of the standard model. He concluded that although conventional dimensional regularisation [15] (DREG) leads to $n=2$, the appropriate choice was $n=4$. This preserves the number of gauge degrees of freedom and hence respects supersymmetry; it corresponds to the use of regularisation by dimensional reduction (DRED) [16]. Supersymmetric theories (which are quite generally free of quadratic divergences [17]) indeed satisfy the equation $\Delta_{1}=0$, with $n=4$ (not $n=2$ ). [En passant, this raises the following issue: imagine that supersymmetry were yet to be discovered. Could one have chanced on it by seeking solutions to the equation $\Delta_{1}=0$ ? Evidently only with the choice $n=4$; that is, by use of a regularisation that preserves the undiscovered symmetry! This is reminiscent of the analysis of finite supersymmetric theories, where finiteness is not manifest if DREG is employed. ${ }^{\star}$ The existence of finite theories can, however, be inferred (from the DREG re-

\footnotetext{
$\star$ For a review and references see [18]
}

sults) by means of coupling constant reparameterisation.]

Use of DRED in a non-supersymmetric context leads, however, to new problems [19]. Consider the infinitesimal gauge transformation on the vector field:

$\delta W_{\mu}=D_{\mu} \alpha=\partial_{\mu} \alpha+\alpha_{\wedge} W_{\mu}$.

With DRED we can separate $W_{\mu}$ into $d$ components $W_{\hat{\mu}}$ and $\varepsilon=4-d$ components $W_{\sigma}$. (3.3) becomes

$\delta W_{\hat{\mu}}=\partial_{\hat{\mu}} \alpha+\alpha_{\wedge} W_{\hat{\mu}}$

$\delta W_{\sigma}=\alpha_{\wedge} W_{\sigma}$.

The $W_{\sigma}$ transform as scalars (and are sometimes called $\varepsilon$-scalars) [16]. This has the effect, for example, that the "gauge" coupling constant of $W_{\sigma}$ to matter fields renormalises differently from the true gauge coupling constant $g$, and the tree $W_{\sigma}^{4}$ interaction is not form-invariant under renormalisation. Most significantly, in the present context, it means that the components of the $W_{\mu}$ self-energy corresponding to the $\varepsilon$-scalars are not protected by gauge invariance from quadratic divergences. Such a divergence indeed occurs at the one loop level; although unphysical, it affects the generalisation to gauge theories of the relationship (2.2). Specifically, we have [7]

$$
\begin{aligned}
\Delta_{2}= & -12 \operatorname{tr}\left(Y_{a} Y_{a}^{*} M M^{*}\right)-6\left[\operatorname{tr}\left(Y_{a} M^{*} Y_{a} M^{*}\right)+\text { c.c. }\right] \\
& +6 U_{a b} \operatorname{tr}\left(Y_{a} Y_{b}^{*}\right)+U_{a b c} U_{a b c}-12 g^{2} U_{a b}\left(\Theta^{2}\right)_{a b} \\
& +(12 n-18) g^{4} \Phi^{T} \Theta^{2} \Theta^{2} \Phi+24 g^{2} \operatorname{tr}\left(t^{2} M M^{*}\right) \\
& +6(n-1) g^{2} \operatorname{tr}\left(Y_{a} Y_{b}^{*}\right)\left(\Theta^{A} \Phi\right)_{a}\left(\Theta^{A} \Phi\right)_{b} \\
& +g^{4}\left[3 T(\Theta)+6(n-2) T(t)-3(n+7) C_{2}(G)\right] \Phi^{T} \Theta^{2} \Phi
\end{aligned}
$$

where $T(R) \delta^{A B}=\operatorname{Tr}\left(R^{A} R^{B}\right)$ and $C_{2}(G) \delta^{A B}=f^{A C D} f^{B C D}$.

As before DRED corresponds to $n=4$ but now DREG corresponds to $n=3$.

It is straightforward to evaluate

$A_{11}=\left[\beta_{U}^{(1)} \cdot \frac{\partial}{\partial U}+\beta_{M}^{(1)} \cdot \frac{\partial}{\partial M}+\beta_{g}^{(1)} \cdot \frac{\partial}{\partial g}\right] \Delta_{1}-\Delta_{1} \cdot \frac{\partial}{\partial U} \beta_{U}^{(1)}$

using the one-loop $\beta$-functions [20]:

$$
\begin{aligned}
16 \pi^{2} \beta_{g}^{(1)}= & {\left[\frac{1}{6} T(\Theta)+\frac{2}{3} T(t)-\frac{11}{3} C_{2}(G)\right] g^{3} } \\
16 \pi^{2} \beta_{U}^{(1)}= & \frac{1}{2} U_{a b} U_{a b}+\frac{3}{2} g^{4}\left(\Phi^{T} \Theta^{A} \Theta^{B} \Phi\right)^{2} \\
& -\operatorname{tr}\left(M M^{*} M M^{*}\right)-3 U_{a}\left(\Theta^{2} \Phi\right)_{a} g^{2} \\
& +\frac{1}{2}\left[U_{a} \operatorname{tr}\left(Y_{a} Y_{b}^{*}\right) \Phi_{b}+\text { c.c. }\right] \\
16 \pi^{2} \beta_{M}^{(1)}= & -3\left\{t^{2}, M\right\} g^{2}+2 Y_{a} M^{*} Y_{a} \\
& +\frac{1}{2}\left(Y_{a} Y_{a}^{*} M+M Y_{a}^{*} Y_{a}\right) \\
& +\frac{1}{2} Y_{a} \Phi_{b} \operatorname{tr}\left(Y_{a} Y_{b}^{*}+Y_{a}^{*} Y_{b}\right) .
\end{aligned}
$$

The result is:

$$
\begin{aligned}
A_{11}= & -12 \operatorname{tr}\left(Y_{a} Y_{a}^{*} M M^{*}\right)-6\left[\operatorname{tr}\left(Y_{a} M^{*} Y_{a} M^{*}\right)+\text { c.c. }\right] \\
& +6 U_{a b} \operatorname{tr}\left(Y_{a} Y_{b}^{*}\right)+U_{a b c} U_{a b c} \\
& -2(n+2) g^{2} U_{a b}\left(\Theta^{2}\right)_{a b}+10(n-1) g^{4} \Phi^{T} \Theta^{2} \Theta^{2} \Phi \\
& +24 g^{2} \operatorname{tr}\left(t^{2} M M^{*}\right)+2(n+5) g^{2} \operatorname{tr}\left(Y_{a} Y_{b}^{*}\right) \\
& +\left(\Theta^{A} \Phi\right)_{a}\left(\Theta^{A} \Phi\right)_{b} \\
& +g^{4}\left[(2 n-1) T(\Theta)+4 T(t)-(n+21) C_{2}(G)\right] \Phi^{T} \Theta^{2} \Phi
\end{aligned}
$$


If we set $n=4$ (corresponding to DRED) in both (3.5) and (3.8), then

$\Delta_{2}-A_{11}=-4 g^{4}\left[T(\Theta)-2 T(t)+2 C_{2}(G)\right] \Phi^{T} \Theta^{2} \Phi$.

This mismatch is a direct consequence of the $\varepsilon$-scalar problem discussed above. Indeed, it is easy to see that $\Delta_{2}-A_{11}$ is proportional to the $\varepsilon$-scalar component of the vector boson self energy. Thus the absence of full fourdimensional gauge invariance engendered by the use of DRED is apparently associated with a breakdown in the relationship between quadratic divergences and scale invariance suggested by (2.2). We could recover (2.2) by modifying $\Delta_{1}$ so as to include in it a term corresponding to the $\varepsilon$-scalar self energy. The condition $\Delta_{1}=0$ would then incorporate the requirement that the $\varepsilon$-scalar self-energy be free of quadratic divergences. This is clearly artificial, however.

Use of DREG as advocated in $[8,12]$ would seem to be indicated, as then there are no $\varepsilon$-scalars to worry about. However, this necessitates setting $n=2$ in (3.8) and $n=3$ in (3.5). Veltman's intuition (and ours) was that this is inappropriate since it misrepresents the number of vector degrees of freedom. Interestingly, there is also no apparent correspondence between $A_{11}$ and $\Delta_{2}$ in that case. Now with logarithmic divergences, one can connect results from different regularisation schemes by means of finite local counterterms; but it is not clear to us how to do this with quadratic divergences. In any event, it is clear that the question of whether a theory can be constrained to be free of quadratic divergences is a very subtle one in gauge theories.

\section{The standard model}

In this section we take the special case of the standard model. We believe it a priori unlikely that the standard model could be rendered free of quadratic divergences to all orders by imposing constraints among a finite number of parameters; nevertheless this topic has generated a certain amount of interest. Therefore we feel it worthwhile discussing the results of the previous section specialised to this case. However we take the DRED case only, for the reasons discussed in the previous section.

In the standard model quadratic divergences arise only in the Higgs self-energy (modulo the $\varepsilon$-scalar problem discussed in Sect. 3). The resulting pole residues are relationships among dimensionless couplings; they can, however, be translated into relationships among particle masses, by multiplying by an appropriate power of the Higgs vacuum expectation value. (We ignore the question of radiative corrections to the masses, and the distinction between renormalised and physical masses).

The result for $\Delta_{1}$ is [1]

$\Delta_{1}=H+3+\tan ^{2} \Theta_{W}-4 T$

* This is true if we work in the symmetric phase of the theory Working in the broken phase, one can instead consider the Higgs tadpole. This seems to us to introduce unnecessary technical complications, however where $H=\frac{m_{H}^{2}}{m_{W}^{2}}, T=\frac{m_{t}^{2}}{m_{W}^{2}}$ and $\Theta_{W}$ is the weak mixing angle. (Contributions from the other quarks and leptons are ignored.)

It is indeed tempting to set $\Delta_{1}=0$, as envisaged originally by Veltman. Fashionable values of $m_{t}$ and $m_{H}$ emerge, for example if $m_{\mathrm{t}} \approx 125 \mathrm{GeV}$ then $m_{\mathrm{H}} \approx 205 \mathrm{GeV}$. It is intriguing that for large $m_{t}, m_{H}$ we have $m_{H}$ slightly less than $2 m_{1}[6]$.

From (3.5) we obtain

$$
\begin{aligned}
\Delta_{2}= & \frac{9}{2} H^{2}+27 H T-54 T^{2}-9 H\left(3+\tan ^{2} \Theta_{W}\right) \\
& -T\left(27-7 \tan ^{2} \Theta_{W}-s\right)+\frac{189}{2} \\
& +45 \tan ^{2} \Theta_{W}+\frac{261}{2} \tan ^{4} \Theta_{W},
\end{aligned}
$$

where

$s=192 \frac{\alpha_{3}}{\alpha} \sin ^{2} \Theta_{W}$,

$\alpha$ and $\alpha_{3}$ are the fine-structure and the strong interaction coupling constants respectively, and from (3.6)

$$
\begin{aligned}
A_{11}= & \frac{9}{2} H^{2}+27 H T-54 T^{2}-9 H\left(3+\tan ^{2} \Theta_{W}\right) \\
& -T\left(27-7 \tan ^{2} \Theta_{W}-s\right)+\frac{27}{2} \\
& +45 \tan ^{2} \Theta_{W}+\frac{109}{2} \tan ^{4} \Theta_{W} .
\end{aligned}
$$

The difference between $\Delta_{2}$ and $A_{11}$ is a consequence of the $\varepsilon$-scalar effect discussed in the previous section.

If we substitute $\Delta_{1}=0$ from (4.1) we obtain

$$
\begin{aligned}
\Delta_{2}= & 126 T^{2}-T\left(324+92 \tan ^{2} \Theta_{W}-s\right) \\
& +216+126 \tan ^{2} \Theta_{W}+144 \tan ^{4} \Theta_{W}
\end{aligned}
$$

and

$$
\begin{aligned}
A_{11}= & 126 T^{2}-T\left(324+92 \tan ^{2} \Theta_{W}-s\right) \\
& +132+126 \tan ^{2} \Theta_{W}+68 \tan ^{4} \Theta_{W} .
\end{aligned}
$$

(4.5) can also be obtained quite simply by taking $\mu \frac{\partial}{\partial \mu}$ of (4.1), and substituting directly the known one-loop standard model $\beta$-functions, and then the equation $\Delta_{1}=0$. This serves as an excellent check of our general results (3.5) and (3.8). We outline this calculation in the Appendix.

Neither $\Delta_{2}=0$ nor $A_{11}=0$ can be achieved for any value of $T$. If we permit ourselves to delete the contribution involving $\alpha_{3}$, then one still cannot achieve $\Delta_{2}=0$, but $A_{11}=0$ for $m_{t} \approx 115 \mathrm{GeV}$ and correspondingly from (4.1) $m_{H} \approx 180 \mathrm{GeV}$. Thus requiring simply that the one loop condition $\Delta_{1}=0$ be scale invariant leads (if the $\alpha_{3}$ term is ignored) to unique predictions for both top and Higgs masses! How seriously should we take this? The mismatch between $A_{2}$ and $A_{11}$ may be resolved by use of a more "suitable" regulator in the calculation of $\Delta_{2}$. The opportunistic neglect of the $\alpha_{3}$ term is harder to justify, and there remains the question of higher order contributions. We are not disposed to attach a great deal of significance to 
our "prediction"; others may differ. It would certainly be interesting, of course, if $m_{t}$ and $m_{H}$ (when known) happen to satisfy $\Delta_{1}=0$; new physics might then enter to ensure $A_{11}=0$. This is Veltman's original mise-en-scène [1], and we are attracted by it, with the following reservation. The obvious tries at "new physics" not affecting $\Delta_{1}$ : for example a gauge boson coupling to the fermions but not the Higgs, or fermions with $S U_{2} \times U_{1}$ quantum numbers but no Yukawa coupling to the Higgs, change $A_{11}$ in such a way as to leave the relations $\Delta_{1}=A_{11}=0$ still incompatible.

\section{Conclusions}

Supersymmetric extensions of the standard model retain interest largely because they render it insensitive to physics from higher mass scales: they are natural. This is founded on the absence of quadratic divergences from supersymmetric theories without $D$-terms. We originally hoped that there might exist nonsupersymmetric theories that were free of quadratic divergences and perhaps natural, but have not been able to provide convincing evidence for this. We did, however, find an interesting correspondence between a theory's quadratic and logarithmic divergences. With dimensional regularisation, this correspondence is clear in non-gauge theories [9].

In the gauge theory case the different results obtained using DRED and DREG raise the question as to whether quadratic divergences, when present, can really be characterised in a meaningful way. One can speculate, for example, that a regulator which preserved both gauge invariance and supersymmetry might remove the discrepancy between $A_{11}$ and $\Delta_{2}$ described in Sect. 3. A possibility worth examining is non-local regularisation [14].

The suggestion that the standard model might, for specific $m_{t}$ and $m_{H}$, be free of quadratic divergences to all orders seem to us most unlikely. Nevertheless we consider the possibility, and conclude that no consistent solution is possible through two loops unless the strong interaction contribution to the Higgs self-energy is ignored. We see no convincing reason to do this: but if it is done, then $m_{t} \approx 115 \mathrm{GeV}$ and $m_{H} \approx 180 \mathrm{GeV}$. Of course it would be most interesting if these turned out to be close to the physical values. [Other approaches to predicting these masses in an unaugmented standard model are, for example, to demand finiteness of the radiative corrections to the masses [21], or to determine them via coupling constant reduction $[5,22]$. We find these approaches also, perhaps, a touch optimistic.]

In conclusion, we feel that the most interesting aspect of this work remains the relationship between the twoloop quadratic divergence and the scale dependence of the one-loop quadratic divergence. Although this relationship appeared to break down at four loops in non-gauge theories [10], it is very possible that this was due to ambiguity in the $\beta$-functions themselves; the fact that a transcendental number ( $(3)$ ) first appears at this loop order is suggestive. In any case further study of quadratic divergences may lead to a better understanding of their structure and role in determining the sensitivity of theories to new physics.

\section{Appendix}

Here we outline the derivation of (4.5) in the standard model direct from Veltman's one loop condition, which in this case is:-

$\Delta_{1}=\lambda+\frac{3}{4} g^{2}+\frac{1}{4} g^{\prime 2}-2 h^{2}=0$

where $g, g^{\prime}, h, \lambda$ are the $S U(2), U(1)$, top, Yukawa and quartic Higgs couplings respectively. Our normalisations are the conventional ones such that

$m_{W}^{2}=\frac{1}{4} g^{2} v^{2}, \quad m_{Z}^{2}=\frac{1}{4}\left(g^{2}+g^{\prime 2}\right) v^{2}$,

$m_{H}^{2}=\lambda v^{2}, \quad m_{t}^{2}=\frac{1}{2} h^{2} v^{2}$

where $v$ is the Higgs vacuum expectation value. Using (A2) it is easy to check that in fact (A1) is identical to (4.1). Taking $\mu \hat{\partial} / \partial \mu$ of (A1), we obtain

$\beta_{\lambda}+\frac{3}{2} g \beta_{g}+\frac{1}{2} g^{\prime} \beta_{g^{\prime}}-4 h \beta_{h}=0$.

The one loop $\beta$-functions for the standard model with three generations and only the $t$-quark Yukawa coupling are:

$$
\begin{aligned}
16 \pi^{2} \beta_{g^{\prime}}= & \frac{41}{6} g^{3}, \quad 16 \pi^{2} \beta_{g}=-\frac{19}{6} g^{3}, \\
16 \pi^{2} \beta_{h}= & \frac{9}{2} h^{3}-\frac{17}{12} g^{\prime 2} h-\frac{9}{4} g^{2} h-8 g_{3}^{2} h, \\
16 \pi^{2} \beta_{\lambda}= & 12 \lambda^{2}-\left(3 g^{\prime 2}+9 g^{2}\right) \lambda+12 \lambda h^{2}-12 h^{4} \\
& +\frac{9}{4}\left(\frac{1}{3} g^{\prime 4}+\frac{2}{3} g^{\prime 2} g^{2}+g^{4}\right)
\end{aligned}
$$

where $g_{3}$ is the QCD coupling constant $\left(g_{3}^{2}=4 \pi \alpha_{3}\right)$.

Substituting (A4) and (A1) in (A3) leads directly to the equation

$A_{11}=0$

where $A_{11}$ is given in (4.5).

The reader is invited to perform the same exercise using the DREG result for $A_{1}$, i.e.

$\Delta_{1}=\lambda+\frac{1}{4} g^{2}+\frac{1}{12} g^{\prime 2}-2 h^{2}$.

As we have explained in the text, however, we attach more significance to the DRED calculation.

\section{References}

1. M. Veltman: Acta Phys. Pol. B12 (1981) 437

2. T. Inami, H. Nishino, S. Watamura: Phys. Lett. B117 (1982) 197

3. N.G. Deshpande, R.J. Johnson, E. Ma: Phys. Lett. B130 (1983) 61; Phys. Rev. D29 (1984) 2851

4. L. Castellani, P. van Nieuwenhuizen: Nucl. Phys. B213 (1983) 305

5. J. Kubo, K. Sibold, W. Zimmermann: Phys. Lett. B220 (1989) 191

6. Y. Nambu: Chicago preprints EFI 88-62, 89-39, 90-46

7. I. Jack, D.R.T. Jones: Phys. Lett. B234 (1990) 321

8. M. Ruiz-Altaba, B. Gonzales, M. Vargas: preprint CERN TH. $5558 / 89$

9. I. Jack, D.R.T. Jones: Nucl. Phys. B342 (1990) 127

10. M.S. Al-sarhi, I. Jack, D.R.T. Jones: Nucl. Phys. B345 (1990) 431

11. C. Wetterich: Z. Phys. C-Particles and Fields 48 (1990) 693

12. M. Capdequi Peryranere, J.C. Montero, G. Moultaka: Phys. Lett. B260 (1991) 138 
13. M. Vargas, J.L. Lucio M.: preprint CINVESTAV/FIS-2191

14. G. Kleppe, R.P. Woodard: Phys. Lett. B253 (1991) 331; G. Kleppe: Phys. Lett. B256 (1991) 431; D. Evens et al.: Phys. Rev. D43 (1991) 499; B.J. Hand, J.W. Moffat: Phys. Rev. D43 (1991) 1896

15. G. 't Hooft, M. Veltman: Nucl. Phys. B44 (1972) 189; C.G. Bollini, J.J Giambiagi: Phys. Lett. B40(1972) 566; J.F. Ashmore: Lett. Nuovo Cimento 4 (1972) 289

16. W. Siegel: Phys. Lett. B84 (1979) 193; D.M. Capper, D.R.T. Jones, P. van Nieuwenhuizen: Nucl. Phys. B167 (1980) 479

17. S. Ferrara, L. Girardello, F. Palumbo: Phys. Rev. D20 (1979) 403

18. D.R.T. Jones: Nucl. Phys. B277 (1986) 153
19. D.R.T. Jones (unpublished) 1979; W. Siegel, P.K. Townsend, P. van Nieuwenhuizen: Proc. 1980 Cambridge meeting on supergravity, ITP-SB-80-65; I. Jack: Phys. Lett. B147 (1984) 405; R. van Damme, G. 't Hooft: Phys. Lett. B150 (1985) 133

20. I. Jack, H. Osborn: Nucl. Phys. B249 (1985) 472

21. R. Decker, J. Pestieau: Lett. Nuovo Cimento 29 (1980) 560; Mod. Phys. Lett. A4 (1989) 2733; I. Hsiu Lee, S.D. Drell: SLACPUB-5423 (Bég Memorial Volume, to be published)

22. M.E. Machacek, M.T. Vaughn: Nucl. Phys. B249 (1985) 70

23. N.-P. Chang: Phys. Rev. D10 (1974) 2706; J. Kubo, K. Sibold, W. Zimmermann: Nucl. Phys. B259 (1985) 331; Phys. Lett. B220 (1989) 185 